\title{
Distribution Category:
}

Mathematics and

Computer Science (UC-405)

\section{ARGONNE NATIONAL LABORATORY \\ 9700 South Cass Avenue \\ Argonne, IL 60439-4801}

ANE-92/11

DE92 017144

ANL-92/11

\section{GenoGraphics for OpenWindows ${ }^{\mathrm{TM}}$}

by

\begin{abstract}
Ray Hagstrom, ${ }^{1}$ George S. Michaels, ${ }^{2}$ Ross Overbeek, Morgan Price, Ronald Taylor, ${ }^{2}$ Kaoru Yoshida, ${ }^{3}$ and Dave Zawada ${ }^{4}$
\end{abstract}

\author{
Mathematics and Computer Science Division
}

April 1992

'High Energy Physics Division. Argonne National Laboratory. Argonne. Il. 60439

${ }^{2}$ Division of Computer Research and Technology. National Institutes of Health. Bethesda, MD 20892

${ }^{3}$ Human Genome Center. Division of Cell and Molecular Biology. Lawrence Berkeley Laboratory. Berkeley. CA 94720

${ }^{4}$ Environmental Assessment and Intormation Sciences. Advanced Computer Applications Cenfer. Argonne National Laboratory. Argonne. IL 60439

This work was supported in part by the Applied Mathemalical Sciences subprogram of the Office of Energy Research. U.S. Department of Energy. under Contract W-31-109-Eng-38. by the Office of Health and Environmental Research. and by the special program in computational molecular biology through the Office of the Director of the Division of Computer Research and Technology. National Institutes of Health. 


\section{Contents}

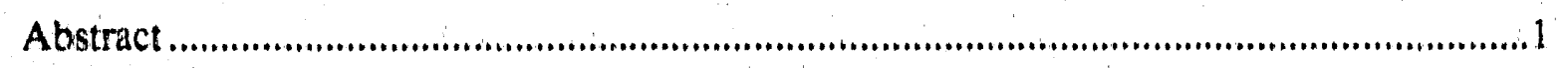

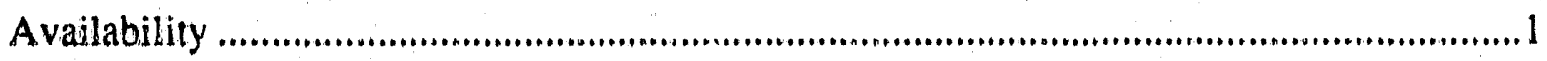

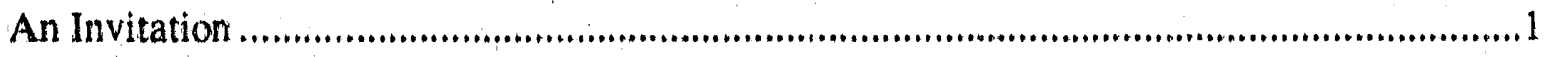

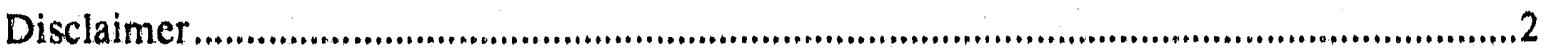

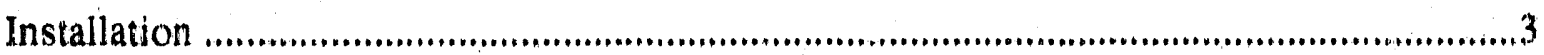

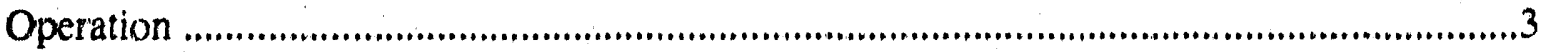

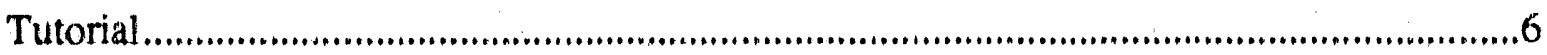

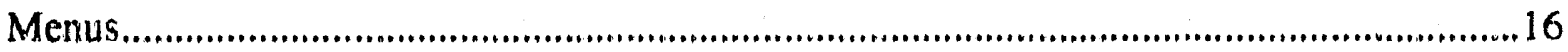

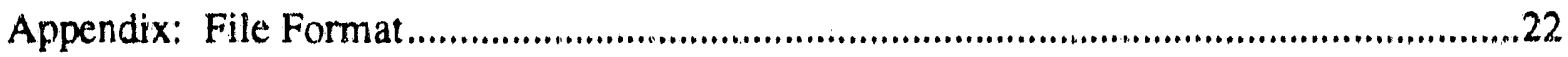

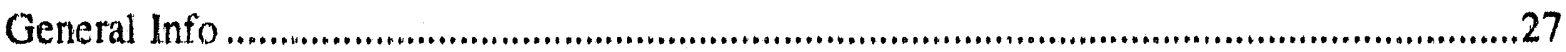




\title{
GenoGraphics for OpenWindows ${ }^{\mathrm{TM}}$
}

\author{
Ray Hagstrom, George S. Michaels, Ross Overbeek, Morgan Price, \\ Ronald Taylor, Kaoru Yoshida, and Dave Zawada
}

\begin{abstract}
GenoGraphics is a generic utility for constructing and querying one-dimensional linear plots. The outgrowth of a request from Dr. Cassandra Smith for a tool to facilitate her genome mapping research. GenoGraphics development has benefitted from a continued collaboration with her. Written in Sun Microsystems's OpenWindows ${ }^{\text {th }}$ environment and the BTOL ${ }^{\text {th }}$ toolkit developed at Argonne National Laboratory, GenoGraphics provides an interactive, intuitive. graphical interface. Its fealures include

- viewing multiple maps simultaneously.

- zooming. and

- querying by mouse clicking.

By expediting plot generation. GenoGraphics gives the scientist more time to analyze data and a novel means for deducing conclustons.
\end{abstract}

\section{Availability}

GenoGraphics is available via anonymous FTP from info.mes an 1 .gov $(140.221 .10 .1)$ in the directory pub/GenoGraphics. We recommend that the README file be downloaded and read first, as it contains additional instructions.

\section{An Invitation:}

We envision GenoGraphics as an evolving program, one that benefits from user feedback. Feel free to keep us abreast of your likes and dislikes, as well as additional capabilities you think should be part of GenoGraphics. As the primary developer of GenoGraphics for OpenWindows ${ }^{\text {tw }}$, please direct such correspondence to Dave Zawada at

EID-900

Argonne National Laboratory

9700 South Cass Avenue

Argonne, Illinois 60439, USA

E-mail: zawada@athens.eid.anl.gov

FAX: (708) 252-512.8 


\section{Disclaimer}

Open Windows ${ }^{\mathrm{m}}$ is a trademark and product of Sun Microsystems, Inc.

BTOL ${ }^{\text {"w }}$ is a product of Argonne National Laboratory and is made available for unrestricted use provided that this legend is included on all tape media and as a part of the software program in whole or part. Users may copy or modify this file without charge but are not authorized to license or distribute it to anyone else except as part of a product or program developed by the user.

THIS FILE IS PROVIDED AS IS, WITH NO WARRANTIES OF ANY KIND INCLUDING THE WARRANTIES OF DESIGN, MERCHANTABILITY, AND FITNESS FOR A PARTICULAR PURPOSE OR ARISING FROM A COURSE OF DEALING, USAGE, OR TRADE PRACTICE.

BTOL $^{n \mu}$ is provided with no support and without any obligation on the part of Argonne National Laboratory to assist in its use, correction, modification, or enhancement.

ARGONNE NATIONAL LABORATORY SHALL HAVE NO LIABILITY WITH RESPECT TO THE INFRINGEMENT OF COPYRIGHTS, TRADE SECRETS, OR ANY PATENTS BY THIS FILE OR ANY PART THEREOF.

In no event will Argonne National Laboratory be liable for any lost revenue or profits or other special, indirect, and consequential damages, even if Sun has been advised of the possibility of such damages. 


\section{Installation}

The present version of GenoGraphics requires a computer running OpenWindows ${ }^{\mathrm{TM}}$. The distribution medium provides the remaining essential files in two directories:

argonne home contains the $\mathrm{BTOL}^{\mathrm{m}}$ toolkit and utilities.

GenoGraphics contains the executable program and sample data files in the maps directory.

Two environment variables also must be set: ARGONNE HOME to the full path to the argonne_home directory, and GMAPHOME to the full path to the GenoGraphics directory. In addition, the user's path must be appended to include the argonne_home/bin directory. To avoid having to repeat this process every time you log in and wish to run GenoGraphics, add the following lines to your $/$.cshrc file:

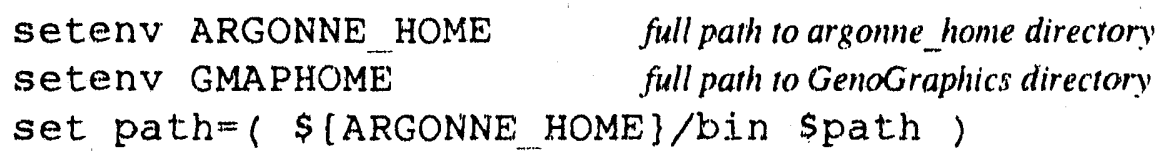

It would be a good idea to log out and log back in to ensure that these environment variables and path additions are in effect.

\section{Operation}

Once you have successfully completed the installation process and entered the OpenWindows ${ }^{\text {"* }}$ environment, GenoGraphics can be launched by typing "gg \&" with in the GenoGraphics directory.

NOTE: If GenoGraphics should become stuck or nonfunctional, you will have to kill the process associated with it. Before the process can be killed, you must determine its process number, which can be found by entering the following command:

$$
\text { ps ugax I grep gg }
$$

In the output from ps, look for line similar to this one:

$$
\text { zawada } 10560 \quad 0.0 \quad 1.292 \quad 376 \text { p3 s 10:06 0:00 gg }
$$

The first number, 10560 in this case, is the process number. To kill the gg process, enter the command

$$
\mathrm{kil1}-9 \text { 1.0560; } \mathrm{nbz}
$$

The program nbz resides in \$ARGONNE_HOME/bin and will remove the image of the "zombied" program from your screen. 
GenoGraphics utilizes the BTOL ${ }^{\mathrm{T}}$ toolkit, which has some distinct features of its own. For instance, its windows may contain any combination of the following icons in their upper comers.

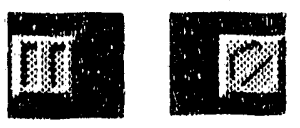

Clicking on the first icon will close a window, its subwindows, and its menu palettes into a single $32 \times 32$ pixel icon. This effect is identical to that achieved by choosing the Hide option from the menu palette. If a window displays this icon, it will always appear in the upper left-hand corner of the window. Clicking on the second icon will dismiss the window without closing it to an icon. In the display window, this dismissal has the same effect as choosing the "Quit" option from the menu palette. If a window displays this icon, it will always appear in the upper right-hand corner of the window.

After being loaded, GenoGraphics will present you with a menu palette and the display window. The display window initially contains the Argonne National Laboratory insignia and asks you to select a dataset. Clicking on the Open option under the File menu will present a scrolling list of the directory's contents in which "gg \&" was typed (see Figure 1).

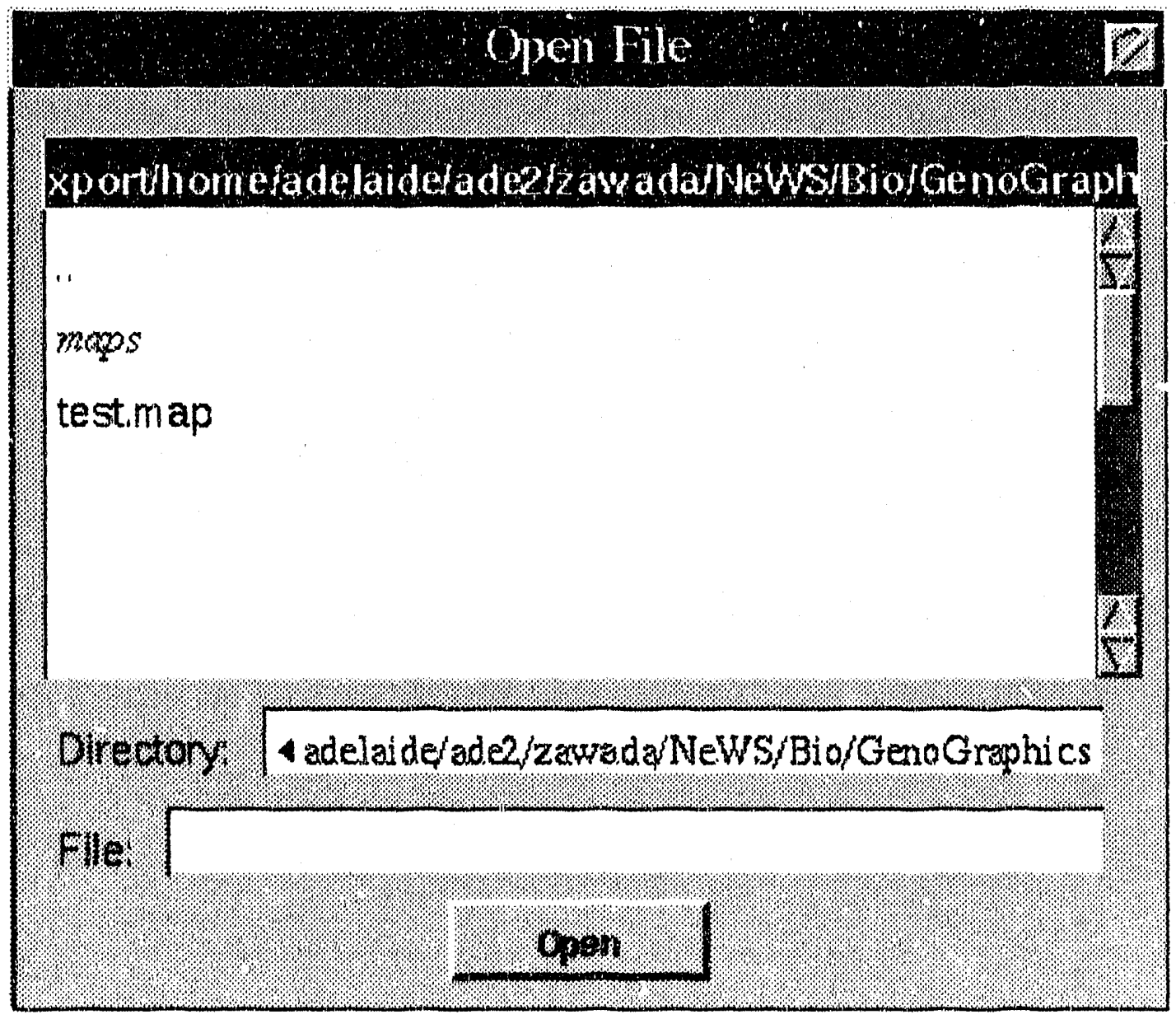

Figure 1: Scrolling directory listing / File selection window 
Items that appear in red, italicized type are other directory names, and items that appear in black are data files. Notice that only those files that end with ".map" are shown in the list. GenoGraphics recognizes files only if they have this extension. Selecting a file and clicking the "Done" button will cause the file to be processed.

GenoGraphics will accept command_line arguments. Thus, the above file-loading procedure could have been accomplished with the command

$$
\text { gg maps/test.map \& }
$$

which would have launched gg in the background and automatically loaded the file "test.map."

Once the file has been read, you will be presented with a checklist of maps that were found in the file, similar to the one in Figure 2 below. Simply click in the box beside each map you would

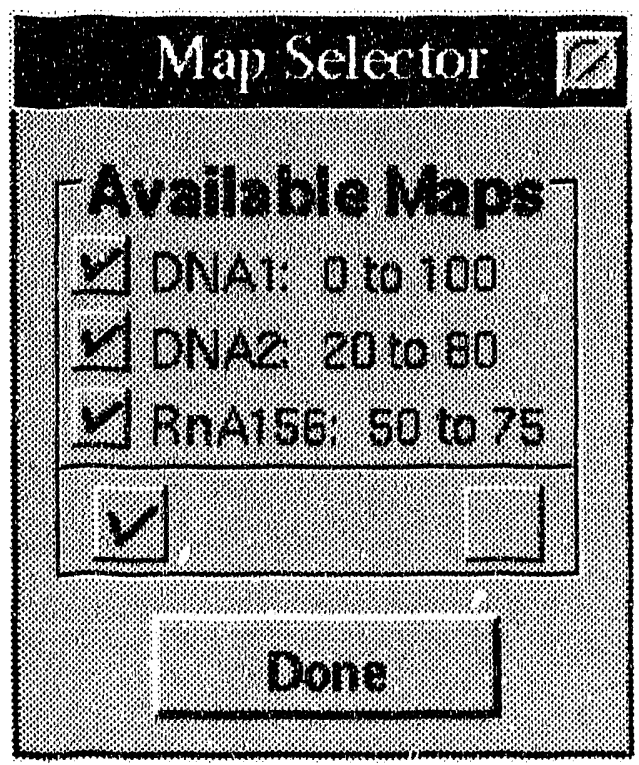

Figure 2: Checklist of available maps for viewing

like to view. Your selections are indicated by a checkmark in the box. Clicking on a checked box will de-select the map and remove the checkmark from the box beside its name. At the bottom of the list, notice the two boxes. Clicking in the box that has the checkmark will select all of the maps in the list. Conversely, clicking in the empty box will de-select all maps in the list. Pressing the "Done" button will fill the display window with the chosen map (s), one below the previous one.

Now, you have access to all of GenoGraphics's querying and viewing features. For a tour of GenoGraphics, read the Tutorial section below. Enjoy! 


\section{Tutorial}

This section provides you with a self-guided tour of GenoGraphics through a hypothetical work session. Follow the directions in the Installation and Operation sections above to get

GenoGraphics up and running. Load the provided sample data file called "test.map." The display window should look like the one in Figure 3. In this picture, the numerical scales above each map are too cluttered. This situation may be remedied in one of two ways. You could simply stretch the window by clicking and dragging in one of the resize controls present at the bottom

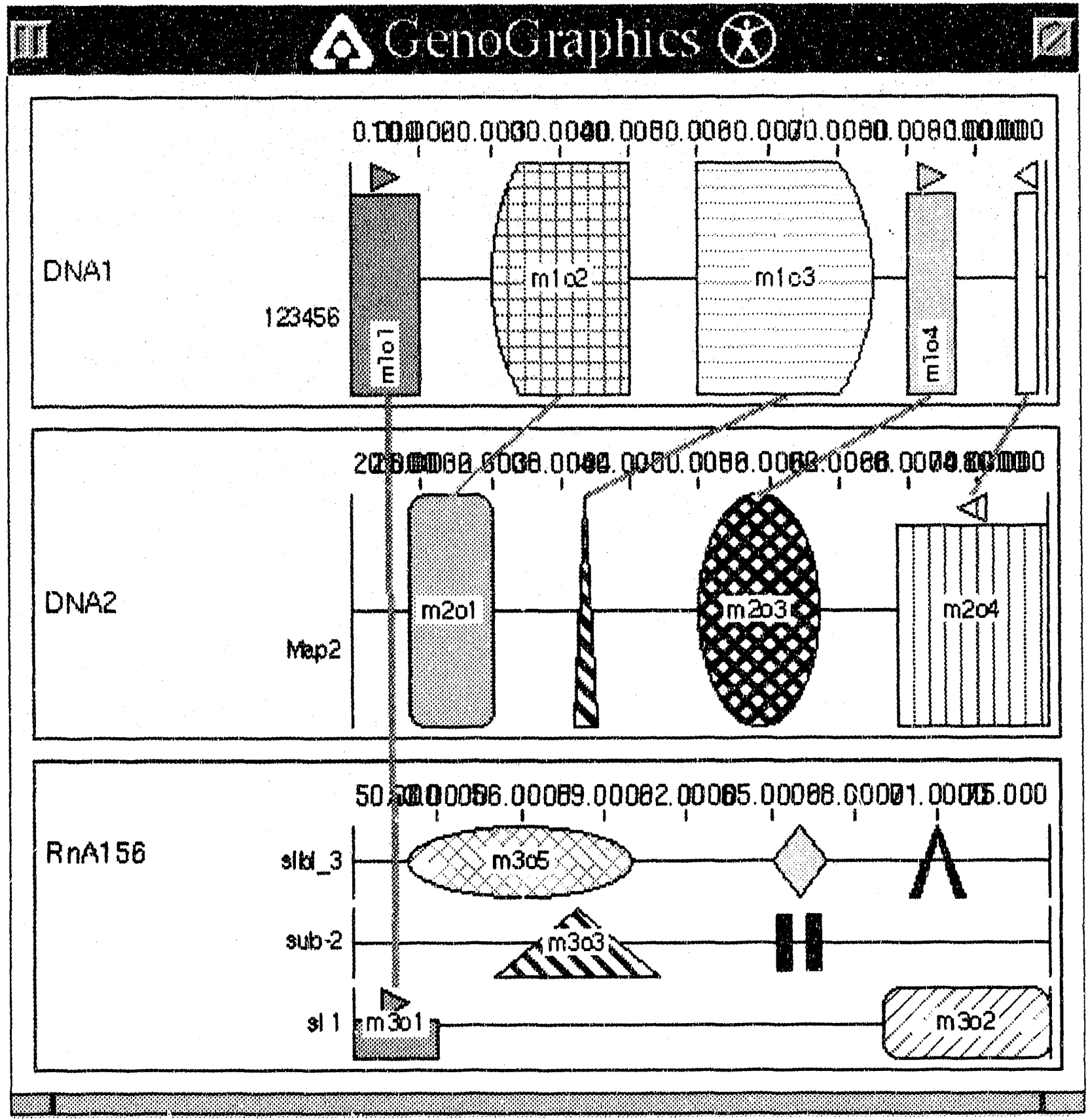

Figure 3: Initial view of display window after loading in the test.map file 
corners of the window. Or, you could select the Set Divisions option from the Scale menu within the Maps menu. Doing so will present the window shown in Figure 4. Next tc the words "Current Map:"

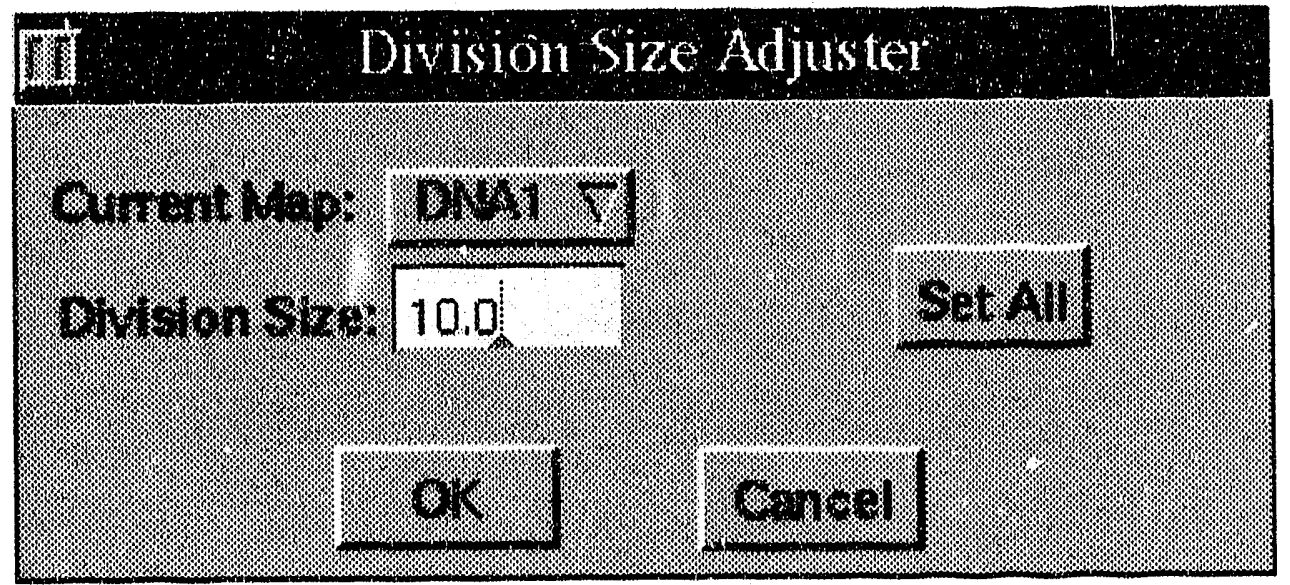

Figure 4: Customization window for map scale

is a popup item. The downward-pointing arrow indicates that clicking on this item will present a list of choices, in this case maps. Selecting a map from the popup item will update the "Division Size:" text field with the current division size setting of that map. Clicking anywhere inside the text field will activate it and enable editing of the value.

Change the "10.0" to "20" and press the "Set All" button followed by the "OK" button. The display window will be updated with all the maps showing scales with divisions every 20 units. A single map's scale may be adjusted by choosing it from the popup item, editing its value, and pressing "OK."

Fixing one problem has revealed another: Each of the three maps has different bounding values. Bounding values may be modified by selecting the Adjust Bounds... option from the Maps menu, which provides the window in Figure 5. Use the "Current Map" popup item to

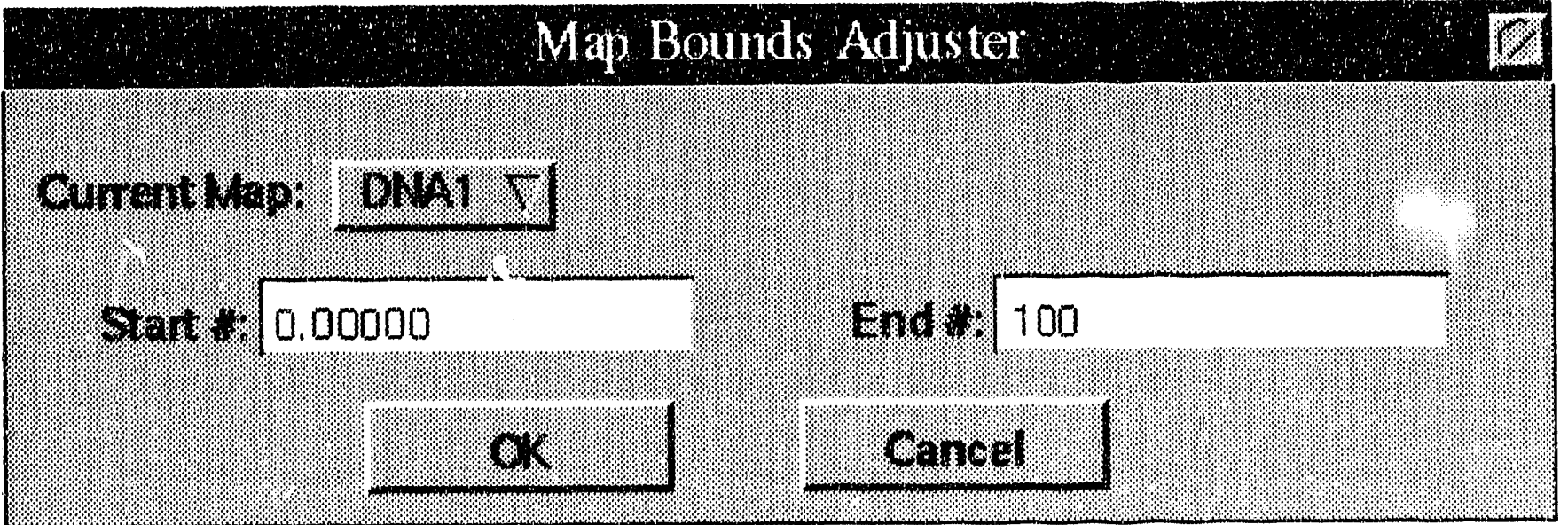

Figure 5: Customization window for map bounding values 
change the start and end bounding values for each map to be "0" and " 100 ," respectively. After you press the "OK" button, the display window will be updated as shown in Figure 6.
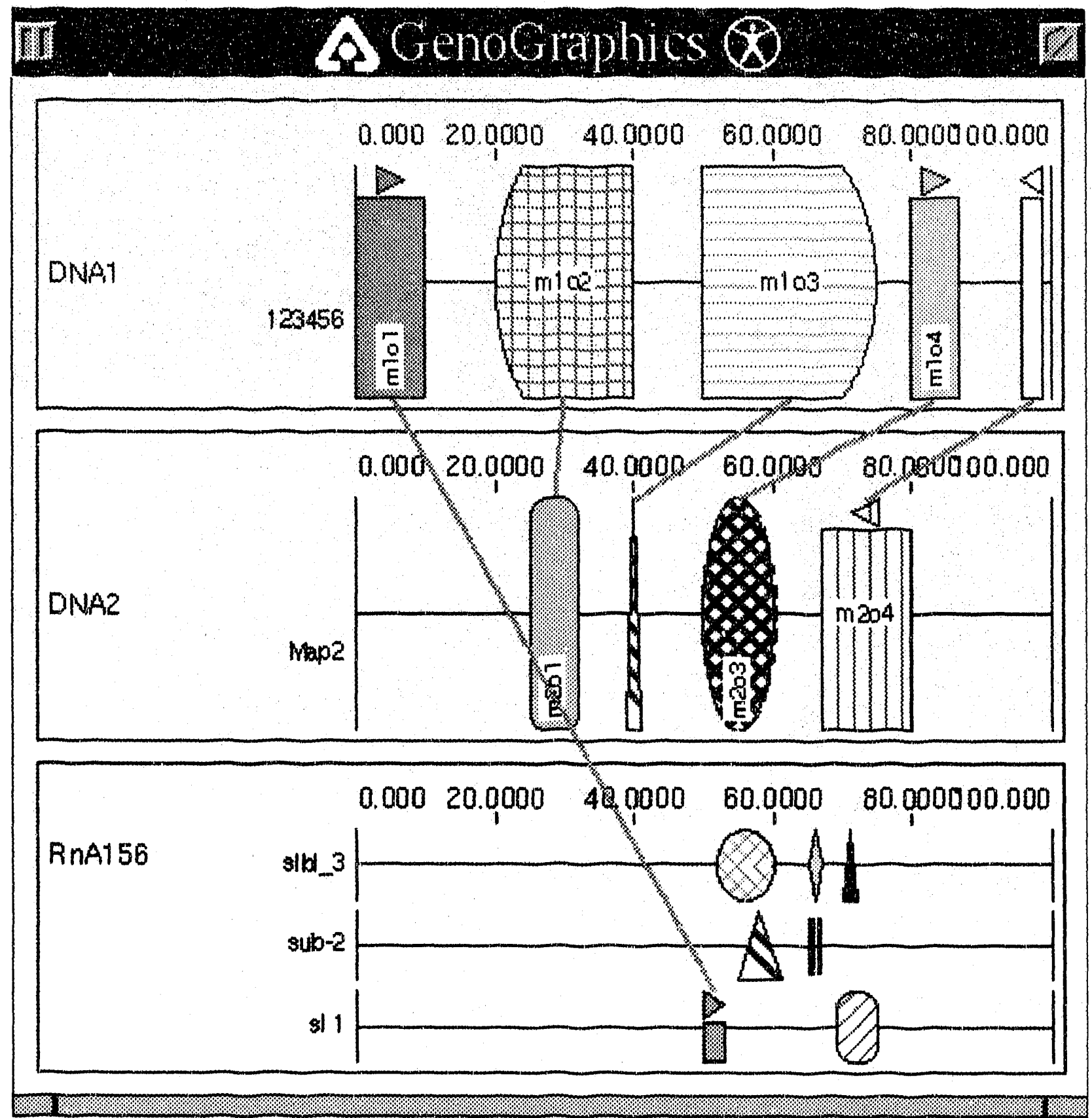

Figure 6: Display window after adjusting the scales and bounding values of each map

Now, that we have uniform map scales and bounding values, let's change the display order of the maps. Choose the Shuffle option from the Maps menu. Notice that when the cursor is placed within the display window, it changes from an arrow to an X, signifying that you should select a map. Click in the map labelled "RnA156." Its border becomes highlighted with a black rectangle that can be dragged vertically inside the display window. Drag and drop this skeleton 
map sver the map currently at the top of the window, the one labelled "DNA1." Upon release of the mouse button, GenoGraphics shuffles the maps into a new order where "RnA156" is first, followed by "DNA1," which is followed by "DNA2." The cursor regains its normal arrow shape to indicate that you are no longer in shuffle mode. Subsequent shuffles may be performed by choosing the Shuffle option.

You've probably realized by now that the "RnA156" map differs from the others, possessing multiple levels or submaps. This feature allows objects that occupy approximately the same coordinate space to be seen unobstructed by placing them on different submaps. You have complete control over which submaps are visible. By default all are visible. Pick the Select SubMaps... option from the Maps menu. Figure 7 shows the submap selection window.

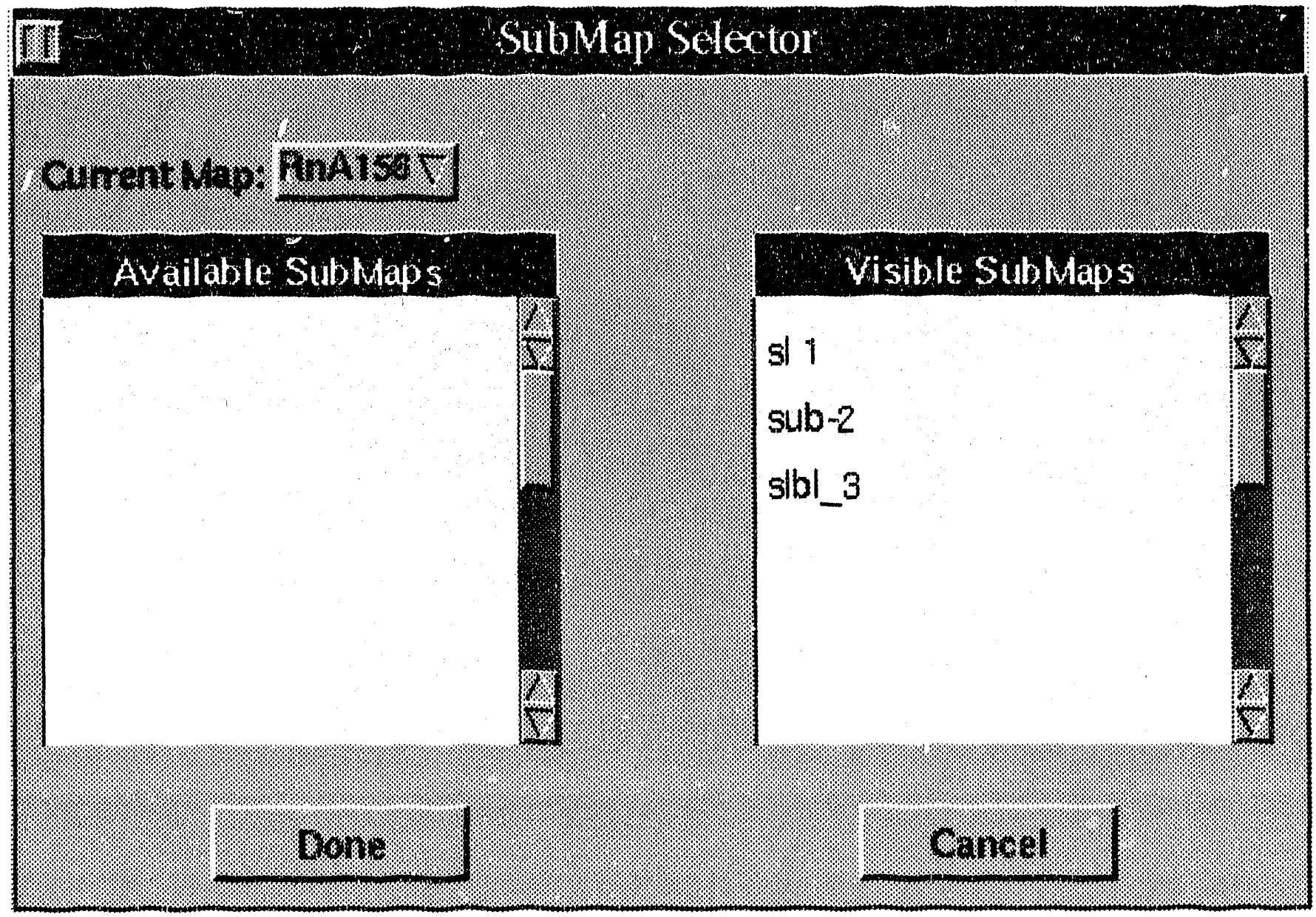

Figure 7: SubMap selection window indicating that all submaps are visible for map "RnA156"

Clicking on a name in the "Visible SubMaps" list will move it to the "Available SubMaps" list and vice versa. At least one submap must be visible; GenoGraphics prevents you from hiding all of a given map's submaps. A list of all maps that possess multiple submaps is available by clicking on the popup item. Pressing the "Done" button will implement any changes. As an exercise, move "sub-2" to the available list. 
Since map "RnA156" now has two visible submaps, compared with one for the others, it would be nice if this map had a little more vertical space and the others a little less. To alter the height of a map, first choose the Resize option from the Maps menu. Notice that the cursor changes from a plain arrow to one with a diamond at its end when placed within tire display window. This change signifies that you are in resize mode.

Clicking inside a map places two black knobs at its top-center and bottom-center. If you click from one map to the next, these knobs follow accordingly. For the first map in the window, only the bottom-center knob will be visible; for the last map in the window, only the top-center knob will be visible. Figure 8 depicts a map with both resize knobs visible.

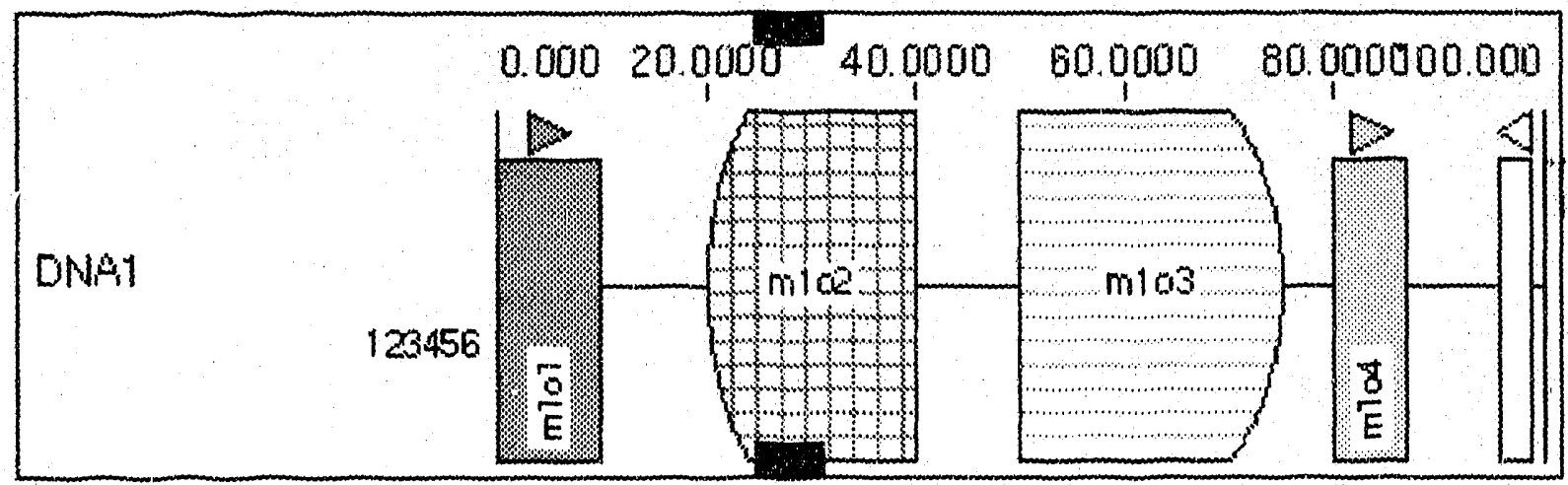

Figure 8: View of a map with resize knobs

To shrink map "DNA1," press and hold the middle mouse button inside the top-center k1sob and drag it downwards. As you drag, notice the gray, rectangular map skeleton that moves with the mouse. This skeleton indicates the new height the map will have when you released the mouse button. You can leave the resize mode by pressing the right mouse button inside either knob.

Once you have successfully shuffled maps, selected submaps, and resized maps as described above, your display window should resemble that of Figure 9. So far, the only features we have discussed pertained to manipulating maps. What type of functionality does GenoGraphics provide for operating on map objects?

All operations pertaining directly to objects are located under the Objects menu. The simplest manipulation is tuming on and off the object labels. The Labels submenu provides for this capability.

Sometimes objects may be too small to display their labels; other times you may know the name of an object, but not its location. To rectify both of these cases, we added the Query... option to the Objects menu. Choosing this option brings up a window similar to the one shown in Figure 10.

At the top of this window is the familiar "Current Map:" popup item which specifies the object set to be queried. If you know the unique name of the object you are trying to locate, enter it into the "Object to Locate:" text field, and press the "Locate" Uutton. GenoGraphics will search 
its database and, after a few seconds, return the starting coordinate of the object in question. If you are uncertain about an object's unique name, press the "S.low List" button at the bottom of the

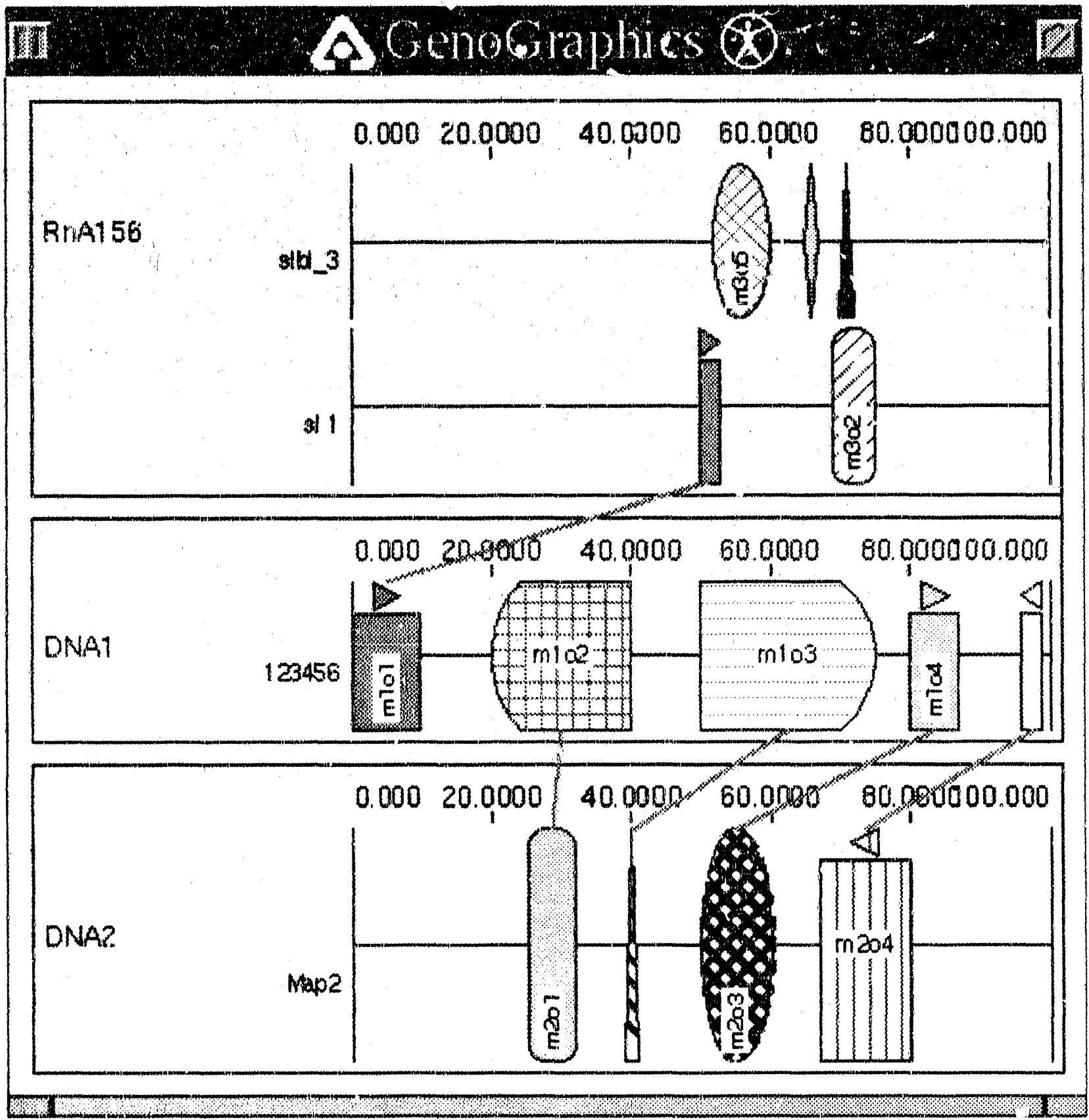

Figure 9: The display vindow after sluffling maps, hiding submaps, and resizing maps

window. GenoGraphics will load the "Object List" scrolling field with the unique names of all the objects belonging to the current map. Clicking on a name in this list will automatically enter it into the "Object to Locate:" text field. As before, press the "Locate" button to determine the starting coordinate for this object. As an exercise, set the current map to be "DNAl, "press the "Show List" button, and instruct GenoGiraphics to find the location of "DNA1lslo3," which is "50." 
In future versions of GenoGraphics, the querying capability will be expanded to facilitate more meaningful inquiries, including conditional and sequence-oriented statements.

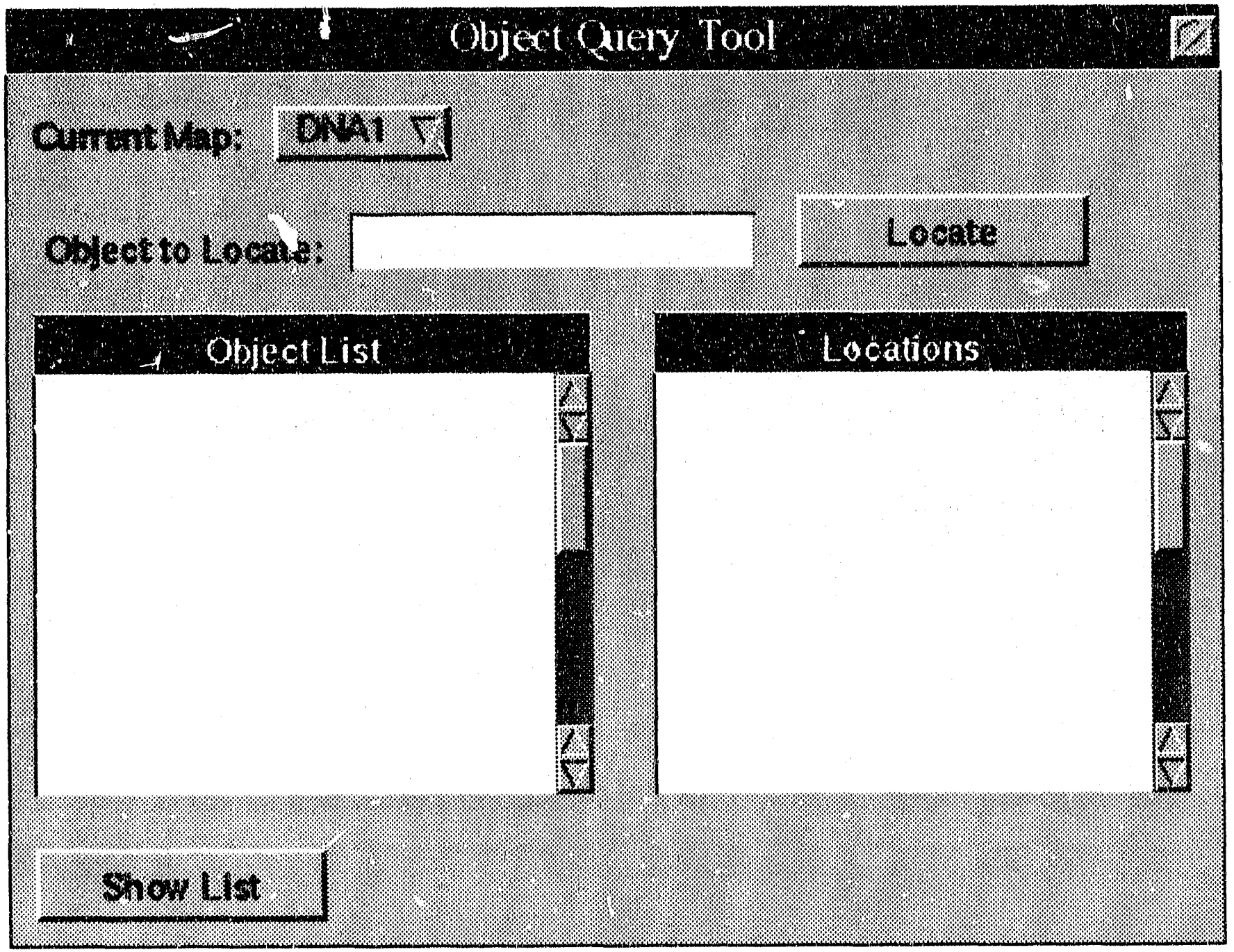

Figure 10: Initial appearance of the Object Query Tool window

On occasion, it may be desirable to view only those objects that lie within a specific range on a given map. The Filter... option in the Objects menu allows you to accomplish such a task. Choosing this option will display a window similar to the one in Figure 11. For the current map, both its bounding values and object filter bounding values are shown. Only the object filter bounds can be edited in this window. You can cycle through all the maps, adjusting their filter bounds as desired. When you are ready to save these changes, press the "OK" button. GenoGraphics will update the display window. Only those objects that lie within the specified range, either completely or partially, will be visible in the updated view.

For example, try specifying object bounds of " 20 " and " 80 " for the "DNA1" map. The display window should resemble the one in Figure 12. Even though object "m104" lies only partially within the specified range, it is still visible. 
Another way to specify that only objects within a ceitain range are visible is to zoom in on that range. Unlike the object filter, which allows you to selectively set a visible object range for

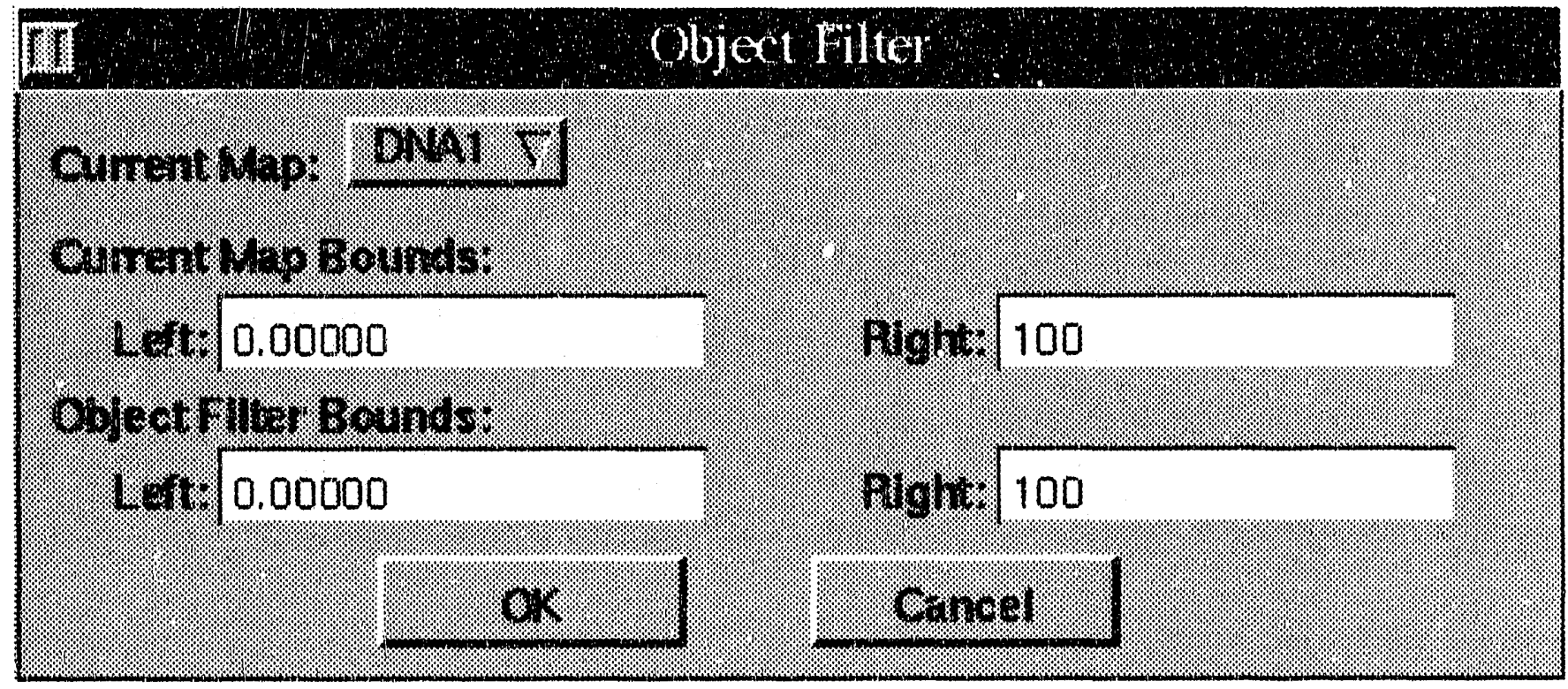

Figure 11: Initial appearance of the Object Filter window

each map, zooming is a global action that affects the visible object range of all the maps. Zooming is illustrated best by example. First, reset the object filter for the "DNA1" map back to " 0 " and "100" so that all of its objects are visible again. Under the Zooming menu, choose the In option. Move the mouse back into the display window, and note the gray vertical line that spans the height of the window. As you drag the mouse, the line follows. You will use this line to mark the boundaries of the zoom range.

Position the mouse so that the gray line passes through " 20 " on the map scales, and click any mouse button. A dashed black line is drawn to mark the location. Repeat the process, except this time position the gray line around "80." A second dashed black line is drawn, and then the entire display window is updated with a magnified view of the " 20 " to " 80 " portion of each map. Choose the In option again, and zoom in from "40" to "60." You can continue to zoom in up to ten times.

The Zooming menu contains two other options, Out and To Top. Out will zoom out to the previous level, and To Top will reverse all zooming and return you to the topmost view of the maps. Before experimenting with either of these options, let's try another feature related to zooming, Overview.

Choose the Overview menu option to display the Overview window, as illustrated in Figure 13. This window contains a miniature version of the overall view of the maps. On top of this image is a black rectangular frame. The portion of the miniature image that lies within this frame corresponds to the current zoom range within the display window. Click and drag the mouse inside this frame to position it over a different section of the global view. Upon releasc of the mouse button, the display window is updated to contain the new section. Note that while the 
position of the zoom range has changed, its size has remained the same. Thus, by repositioning the frame, you can pan the entire length of the maps while retaining the current zoom range.

The Overview window is dynamic as long as it is visible. Subsequent zooming operations cause the Overview frame to be updated accordingly. Take some time, now, to experiment with the zooming and overview fearures of GenoGraphics.
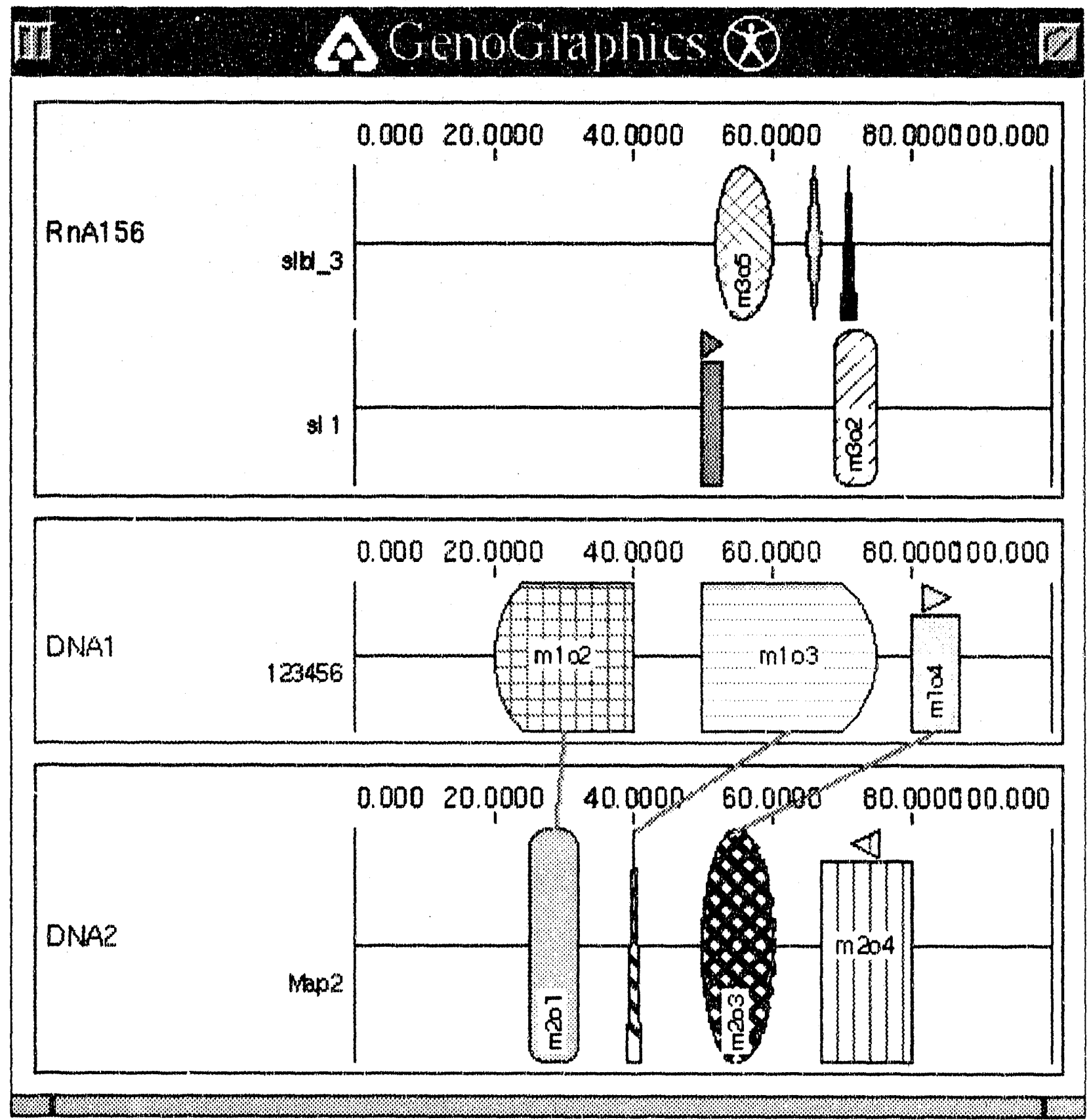

Figure 12: View of the display window after setting the object filter for map "DNAl" 10 " 20 " and " 80 " 


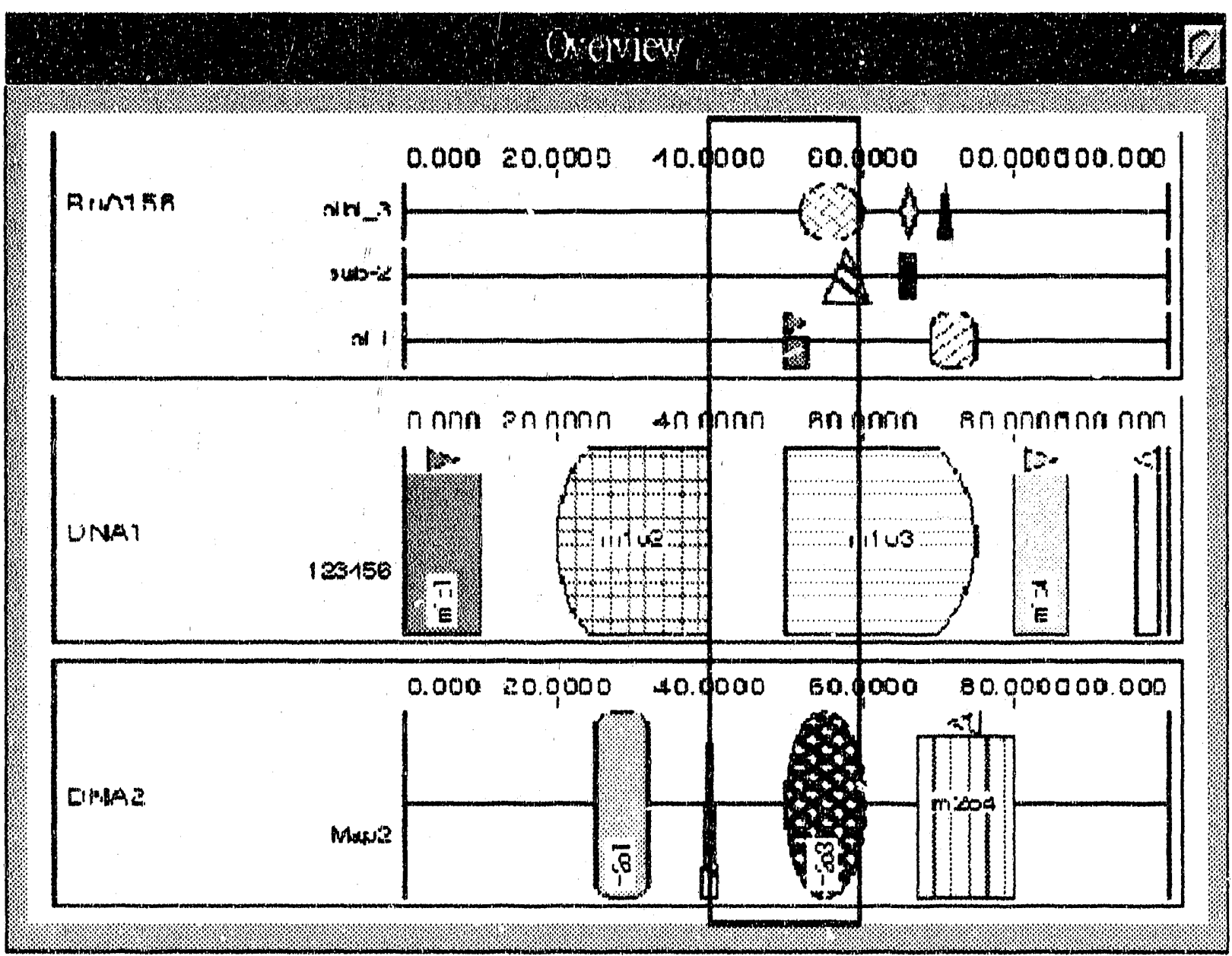

Figure 13: View of the Overview window after two zoom-ins

Two additional features not found in the menus simply involve mouse clicks. Pressing the left mouse button near the label of a map will bring up a window containing background information pertaining to that map. Likewise, pressing the left mouse button on any object will display a window containing background information on that object. The other feature is object relocation. Clicking and dragging the mouse while holding down the middle mouse button inside of an object allows you to reposition that object anywhere on the map.

That concludes our tour of GenoGraphics. The best way to build competency with a piece of software is to play with it. So, get started today! 


\section{Menus}

This section discusses all of the menu options as they appear in the menu palette illustrated below. The arrows that appear to the right of some menu items indicate that a submenu of options is available that apply to that particular category.

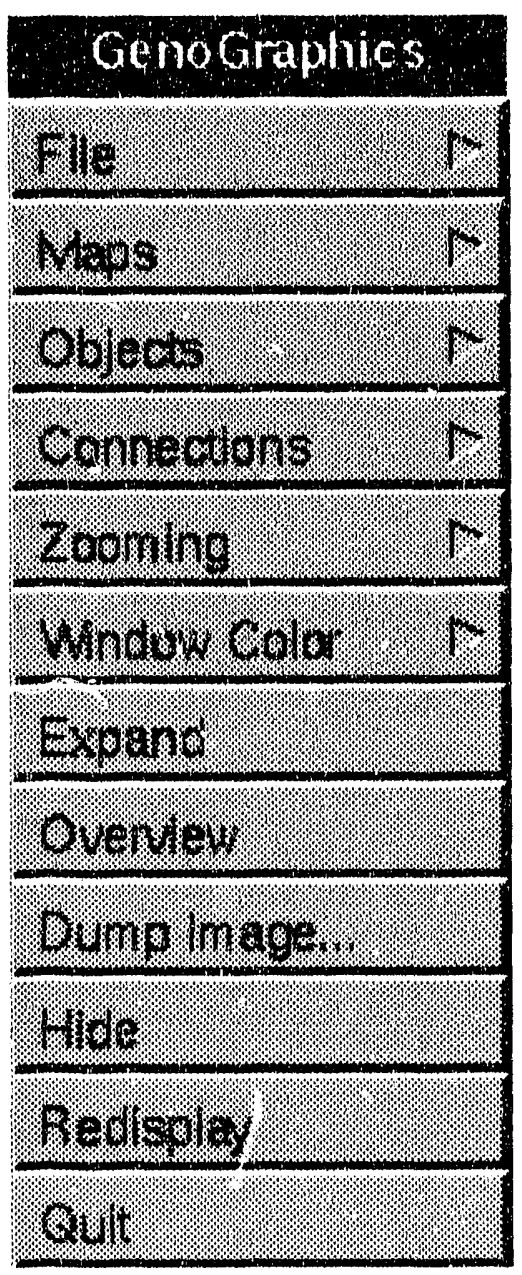


File

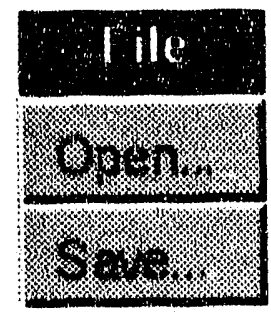

Open...

Presents window containing a scrolling list of the current directory contents (see Figure 1). Directory names appear in red, while GenoGraphics data files (i.e., files with the ".map" extension) appear in black. The full path to the current directory is shown in the text firdd below the scrolling list, as is the currently selected data file. Clicking on a directory name or file name in the scrolling list updates the respective text field. Directory and/or data filenames may be entered directly into the text fields. In any event, pressing the "Done" button will load in the specified file.

Save...

Presents a window similar to Open... above, except its purpose is to obtain directory and file names in which to store the data. This option is disabled in version 1.0alpha.

\section{Maps}

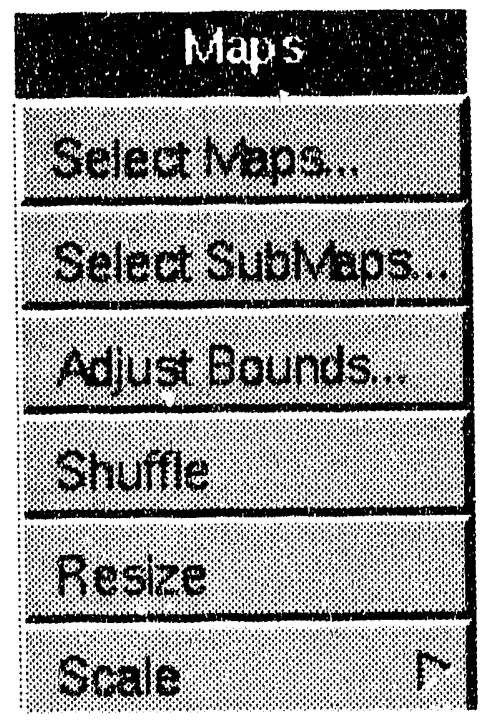

Presents a window with a checklist of maps that are available for display (see Figure 2). This list is the same one that appears after GenoGraphics opens and reads a file. This option allows the visible maps in the display window to be changed at any time. 
Select Submaps...

Presents a window with two scrolling lists entitled "Available" and "Visible," indicating which submaps of a map are visible at any given time (see Figure 7). Sometimes a map contains so many objects that it is too cluttered to discern one object from another. To remedy this situation, GenoGraphics supports the notion of submaps (see the Appendix: File Format) which allows objects to be spread out across multiple levels, similar to notes on a music staff. Clicking on a submap name in either list moves it to its counterpart, thus allowing submaps to be hidden or shown, as desired.

\section{Adjust Bounds...}

Presents a window containing text items that allow the redefinition of the bounding values of any available map (see Figure 5 ). GenoGraphics does not require that every map within one of its data files have the same bounding values. For comparison purposes, however, this feature may be desirable.

\section{Shuffle}

Changes the cursor to a "X." Clicking inside a map with this cursor will display a skeleton image of that map, which may be dragged vertically. The location where this skeleton is dropped will determine where the map will be inserted among the other visible maps. Thus, the order of maps may be altered to preference.

\section{Resize}

Changes the cursor to an arrow with a diamond at its end. Clicking inside a map with this cursor will display two resize knobs the top-center and bottom-center of the map (see Figure 8). The height of the map may be adjusted by clicking and dragging the mouse with the middle mouse button held down inside either knob. Pressing the right mouse button inside either knob exits from the resize mode.

\section{Scale}

Presents a window above each map that allows the customization of any map's scale (see Figure 4). By default, the scale contains ten divisions. 
Objects

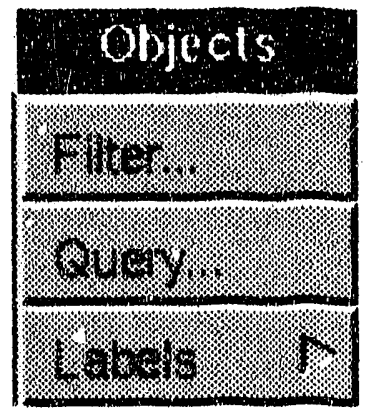

Filter...

Presents a window that allows ranges to be specified for a given map (see Figure 11). Only those objects that lie within the specified range for that map are visible.

Quen:...

Prese ats a window that allows the location of any object to be found on any map (see Figure 10). After a map is selected to search, all of the labels of its objects are placed in a scrolling list. Clicking on a name and pressing the "Locate" button will display its numerical location. This feature is still in the early stages of development and eventually will become a more robust querying utility.

\section{Labels}

Presents another menu with the options On and Off that allow all object labels to be turned on or off.

\section{Connections}

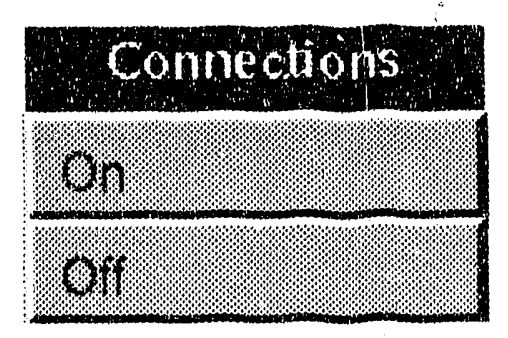

Presents another menu with the options On and Off that allow all connections between objects to be turned on or off. 


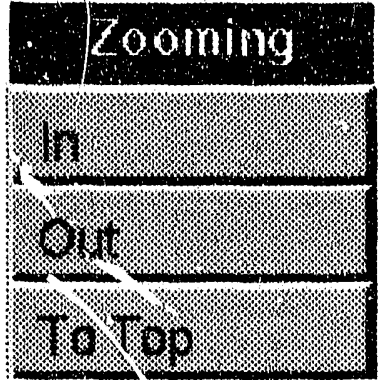

In

'Tells GenoGraphics you would like a magnified view of a specific region. This region is specified by two successive mouse clicks. Each click causes a dashed vertical line to be drawn across all visible maps indicating one of the bounds for the zoom region. After the second click, the display window is updated to show the contents of the zoom region. You can continue to zoom in up to a total of ten times.

Out

Performs the opposite of In above.

To Top

Restores the display window to the highest level view of the maps. In other words, this option reverses all previous zoom-ins.

\section{Window Color}

Presents a palette of optional window colors.

\section{Expand/Unexpand}

Resizes the display window to fill the entire screen or restores it to its previous dimensions.

\section{Overview}

Displays a window containing a miniature version of a global view of all the maps (see Figure 13). Inside this window is a black rectangular frame that outlines the current zoom range of the display window. Clicking and dragging the mouse within the frame repositions it. Thus, panning of the global view is possible while maintaining the current zoom range setting.

\section{Dump Image...}

Creates a screen dump (i.e., raster file) of the display window.

\section{Hide}

Closes all menus and windows into one icon. 


\section{Redisplay}

Refreshes the display window.

Quit

Exits GenoGraphics. 


\section{Appendix: File Format}

This section provides a complete description of the file format required by GenoGraphics. Relevant examples are included that were excerpted from the test.map file used in the Tutorial section.

An input file is composed of two main sections:

a. A set of maps, each of which has the form

MAPBEGIN

MAPEND

b. A set of optional connections, one per line, grouped using

CONNBEGIN

CONNENI

A map has the following format:

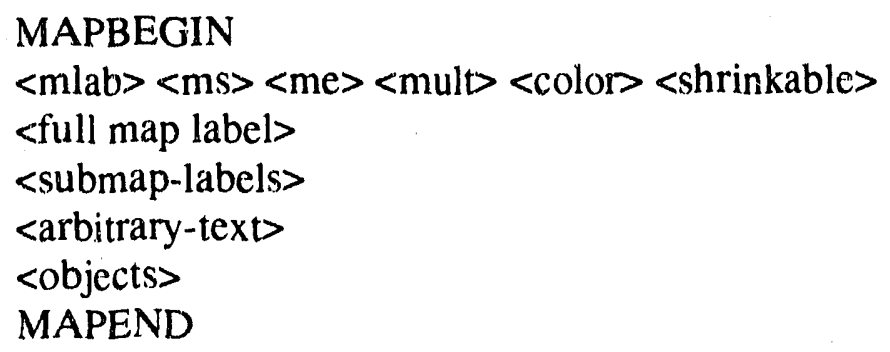

$<$ mlab $>$

$<\mathrm{ms}>$

$<$ me $>$

$<$ mult $>$

$<$ color $>$

$<$ shrinkable>

$<$ full map label>

$<$ submap-labels>

$<$ arbitrary-text> a unique abbreviation for a map id (6-character max.)

lowest coordinate on the map highest coordinate on the map number of submaps (the multiplicity of the map) color of the border and label on the map TRUE or FALSE (for future features) up to 60 characters, including white space 1 per line (6-char max. -- abbreviation) one line containing the number of lines that follow, and then the lines of arbitrary text 
<objects> Each object is of the following form:

$<$ label $><$ unique-id $><$ submap-number $><$ star $><$ end $><$ shape $><$ fill $><$ color $>$ $<$ arbitrary-text $>$

All objects within a given map are sorted according to submap number and then according to start value:

$\begin{array}{lll}\text { object \# } & \text { submap\# } & \text { start value } \\ 1 & 1 & 88 \\ 2 & 1 & 137 \\ 3 & 1 & 1492 \\ 1 & 2 & 1066 \\ 2 & 2 & 1776 \\ 1 & 3 & 101 \\ 2 & 3 & 555 \\ 3 & 3 & 1234\end{array}$

$<$ label $>$

$<$ unique-id>

<submap-number>

$<$ start-

$<$ <end>

$<$ shape>

$<$ fill $>$

$<$ colors

$<$ arbitrary-text $>$ 10-char max. length

unique alphanumeric string of the form

$<$ mlab $>1 \mathrm{~s} \%$ do\%d

with the integer fields identifying

submap number [1..n_submaps_this_map], object number [1..n_objects_this_submap].

map level object goes or $(>=1)$

starting coordinate

ending coordinate

string (see Geneial Info below)

string (see General Info below)

string (see General Info below)

as before, 1 line of count of lines, followed by lines 
EXAMPLE 1: Creation of map "DNA2" which has only one submap

\section{MAPBEGIN}

DNA2 $2,0 \quad 801$ blue FALSE

Full Map Label for Map: >DNA2<

Map2

1

This is Map DNA2

m201 DNA2|s101 12532 roundrect solid yellow-green 0

m202 DNA2|siu2 13941 triangle backslash magenta 2

Iinel of arbitrary text Second line of text

m203 DNA2|s103 150 oval dblslash burgundy 1

Sample Text

m2o4 DNA2|s104 16780 leftarrow vbars steel-blue 0

MAPEND 
EXAMPLE 2: Creation of map "RnA156" which has three submaps

MAPBEGIN

RnA156 50752 black FALSE

Ful1 Map Label for Map: - $>$ RnA156<--

s1. 1

sub- 2

sibl 3

1

Miscellaneous map information

m301 RnA156/s101 15053 rightarrow solid cyan

1

Level. 1

m302 RnA156/s102 16975 roundrect thinslash magenta

3

Level 1 .

linel of arbitrary text

second line of text

m303 RnA156/s20325561 triangle backslash pink

1

Leve 12

m304 RnA156/s204265 67 twobar solid blue

1.

Level 2

m305 RnA $156 / s 30535260$ oval thindblslash green-blue 2

Level 3

No Additional Info Available

m306 RnA156/s306 36567 diamond solid yellow

0

m307 RnA156/5307 37072 caret solid magenta

0

MAPEND 
Connections occur one per line, with the following format:

$<$ mlab $><$ unique-id $><$ mlab $><$ unique-id $><$ direction $>$

$<$ mlab $>$

$<$ <unique-ids

label of parent map

$<$ direction>

unique id of the object

an integer reserved for future use. Present values for $<$ direction $>$ are

0 Undirected connection between objects

1 Arrow points from first to second object

2 Arrow points from second to first object

3 Double-headed arrow between objects

EXAMPLE 3: Creation of connections

\section{CONNBEGIN}

DNA1 DNA1|s101 RnA156 RnA156|s1010

DNA1 DNA1/s102 DNA2 DNA2|s101 0

DNA1 DNA1|s 103 DNA2 DNA2|s102 0

DNA1 DNA1|s104 DNA2 DNA2|s 1030

DNA1 DNA1|s105 DNA2 DNA2|s 1040

CONNEND 
General Info

Valid Colors are

$\begin{array}{llllll}\text { black } & \text { dark-green } & \text { green-blue } & \text { magenta } & \text { red } & \text { yellow-green } \\ \text { blue } & \text { flesh } & \text { grey } & \text { olive } & \text { rust } & \text { yellow-orange } \\ \text { brown } & \text { grape } & \text { lite-blue } & \text { orange } & \text { steel-blue } & \\ \text { burgundy } & \text { gray } & \text { lite-green } & \text { pink } & \text { white } & \\ \text { cyan } & \text { green } & \text { lite-yellow } & \text { purple } & \text { yellow } & \end{array}$

Valid Shapes are

$\begin{array}{lllll}\begin{array}{l}\text { caret } \\ \text { diamond } \\ \text { twobar }\end{array} & \begin{array}{l}\text { ldee } \\ \text { leftarrow }\end{array} & \begin{array}{l}\text { oval } \\ \text { rdee }\end{array} & \begin{array}{l}\text { rectangle } \\ \text { rightarrow }\end{array} & \begin{array}{l}\text { roundrect } \\ \text { triangle }\end{array} \\ & & & & \end{array}$

Valid FillPatterns are

$\begin{array}{lllll}\text { backslash } & \text { dblslash } & \text { slash } & \text { thinbackslash } & \text { thinslash } \\ \text { checker } & \text { hbars } & \text { solid } & \text { thindblslash } & \text { vbars }\end{array}$




\section{Distribution for ANL-92/11}

Interral:
J. M. Beumer (100)
F. Y. Fradin
R. T. Hagstrom (25)
R. A. Overbeek (25)
G. W. Pieper
M. Price (25)
R. L. Stevens
C. L. Wilkinson
D. G. Zawada (25)

\section{ANL Patent Department \\ ANL Contract File \\ TIS Files (3)}

\section{External:}

DOE-OSTI, for distribution per UC-405 (58)

ANL Libraries

Manager, Chicago Operations Office, DOE

Mathematics and Computer Science Division Review Committee:

W. W. Bledsoe, The University of Texas, Austin

P. Concus, Lawrence Berkeley Laboratory

E. F. Infante, University of Minnesota

M. J. O'Donnell, University of Chicago

D. O'Leary, University of Maryland

R. E. O’Malley, Rensselaer Polytechnic Institute

M. H. Schultz, Yale University

J. Cavallini, Department of Energy - Energy Research

F. Howes, Department of Energy - Energy Research

G. Michaels, National Institutes of Health, Bethesda, MD (25)

R. Taylor, National Institutes of Health, Bethesda, MD (25)

K. Yoshida, Lawrence Berkeley Laboratory, Berkeley, CA (25) 

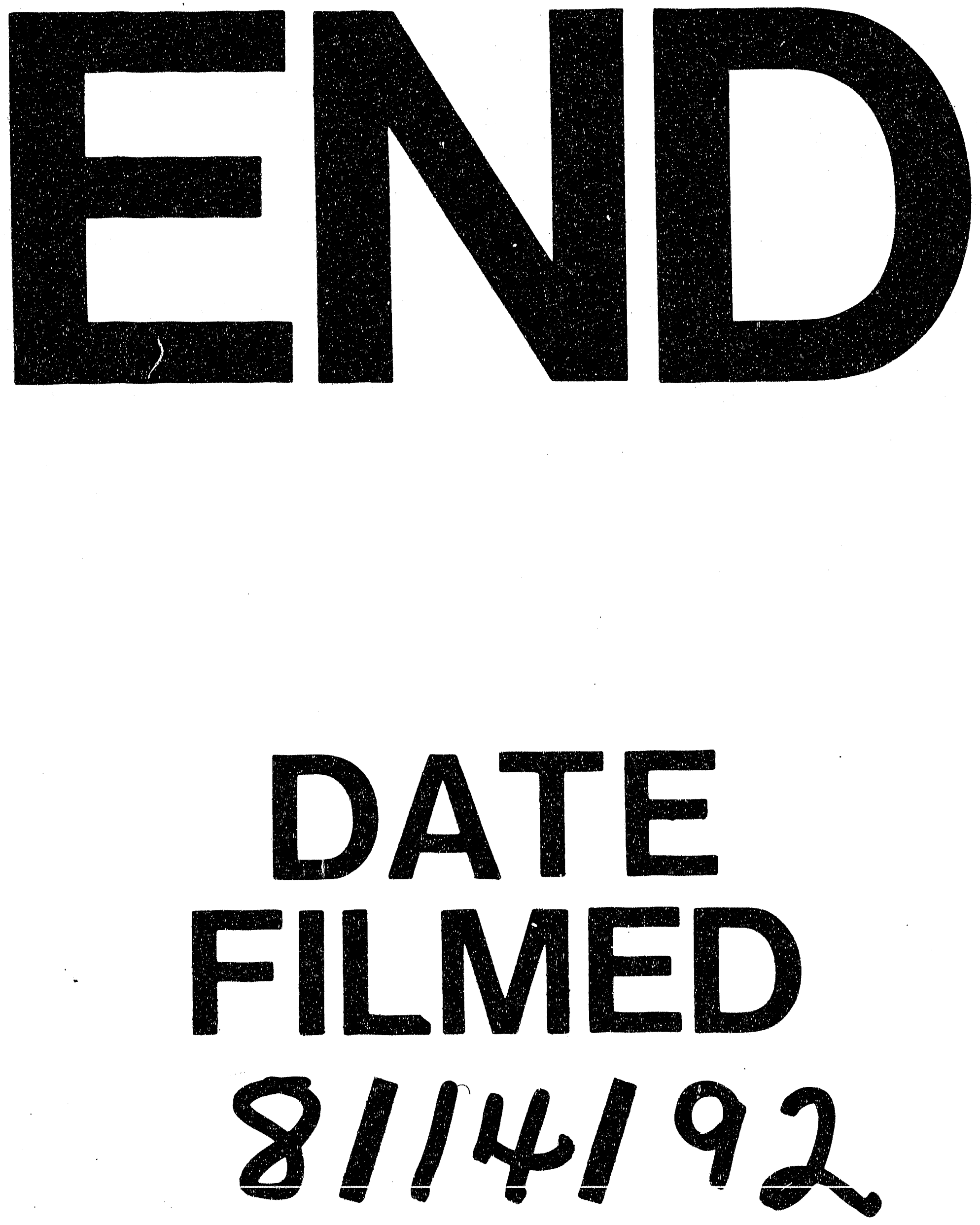
